\title{
Article \\ Aberrant Methylation of SLIT2 Gene in Plasma Cell-Free DNA of Non-Small Cell Lung Cancer Patients
}

\author{
Yujin Kim ${ }^{1}{ }^{\oplus}$, Bo Bin Lee ${ }^{1}\left(\right.$ ) , Dongho Kim ${ }^{1} \oplus$, Sang-Won Um ${ }^{2}$, Joungho Han ${ }^{3}$, Young Mog Shim ${ }^{4}$ \\ and Duk-Hwan Kim ${ }^{1, *}$
}

check for updates

Citation: Kim, Y.; Lee, B.B.; Kim, D.; Um, S.-W.; Han, J.; Shim, Y.M.; Kim, D.-H. Aberrant Methylation of SLIT2 Gene in Plasma Cell-Free DNA of Non-Small Cell Lung Cancer Patients. Cancers 2022, 14, 296. https:// doi.org/10.3390/cancers14020296

Academic Editors: Giuseppe Fanelli, Pier Vitale Nuzzo and Edoardo Francini

Received: 15 November 2021

Accepted: 4 January 2022

Published: 7 January 2022

Publisher's Note: MDPI stays neutral with regard to jurisdictional claims in published maps and institutional affiliations.

Copyright: (c) 2022 by the authors. Licensee MDPI, Basel, Switzerland. This article is an open access article distributed under the terms and conditions of the Creative Commons Attribution (CC BY) license (https:// creativecommons.org/licenses/by/ $4.0 /)$.
1 Department of Molecular Cell Biology, School of Medicine, Sungkyunkwan University, Suwon 440-746, Korea; yujin0328@hanmail.net (Y.K.); whitebini@hanmail.net (B.B.L.); jindonghao2001@hotmail.com (D.K.)

2 Department of Internal Medicine, Samsung Medical Center, School of Medicine, Sungkyunkwan University, Seoul 135-710, Korea; sangwon72.um@samsung.com

3 Department of Pathology, Samsung Medical Center, School of Medicine, Sungkyunkwan University, Seoul 135-710, Korea; joungho.han@samsung.com

4 Department of Thoracic and Cardiovascular Surgery, Samsung Medical Center, School of Medicine, Sungkyunkwan University, Seoul 135-710, Korea; youngmog.shim@samsung.com

* Correspondence: dukhwan.kim@samsung.com

Simple Summary: Despite significant advances in the detection, prevention, and treatment of lung cancer, the prognosis of the patients is still very poor due in part to micrometastasis of cancer cells to surrounding tissues at the time of diagnosis. Therefore, identifying biomarkers for early detection of lung cancer is very important for prolonging the lifespan of patients with lung cancer. The methylation statuses of SLIT1, SLIT2, SLIT3 genes were analyzed in bronchial washing, bronchial biopsy, sputum, tumor and matched normal tissues, or plasma samples obtained from a total of 208 non-small cell lung cancer (NSCLC) patients and 121 cancer-free patients to understand the feasibility of the genes as biomarkers for early detection and survival prediction of NSCLC. The present study suggests that aberrant methylation of SLIT2 in plasma cell-free DNA might be a potential biomarker for the early detection and prognosis prediction of NSCLC patient.

Abstract: This study aimed to understand aberrant methylation of SLITs genes as a biomarker for the early detection and prognosis prediction of non-small cell lung cancer (NSCLC). Methylation levels of SLITs were determined using the Infinium HumanMethylation450 BeadChip or pyrosequencing. Five CpGs at the CpG island of SLIT1, SLIT2 or SLIT3 genes were significantly (Bonferroni corrected $p<0.05)$ hypermethylated in tumor tissues obtained from 42 NSCLC patients than in matched normal tissues. Methylation levels of these CpGs did not differ significantly between bronchial washings obtained from 76 NSCLC patients and 60 cancer-free patients. However, methylation levels of SLIT2 gene were significantly higher in plasma cell-free DNA of 72 NSCLC patients than in that of 61 cancer-free patients ( $p=0.001$, Wilcoxon rank sum test). Prediction of NSCLC using SLIT2 methylation was achieved with a sensitivity of $73.7 \%$ and a specificity of $61.9 \%$ in a plasma test dataset $(N=40)$. A Cox proportional hazards model showed that SLIT2 hypermethylation in plasma cell-free DNA was significantly associated with poor recurrence-free survival (hazards ratio $=2.19$, $95 \%$ confidence interval $=1.21-4.36, p=0.01$ ). The present study suggests that aberrant methylation of SLIT2 in plasma cell-free DNA is a valuable biomarker for the early detection of NSCLC and prediction of recurrence-free survival. However, further research is needed with larger sample size to confirm results.

Keywords: lung cancer; SLIT; methylation; plasma; biomarker

\section{Introduction}

Lung cancer is one of most common causes of cancer-related death worldwide. Despite significant advances in the prevention, detection and treatment of lung cancer over the 
past 20 years, the prognosis of patients with lung cancer is still extremely poor, with the five-year overall survival rate remaining less than $20 \%$ [1]. The prognosis of cancer patients is usually determined by disease stage and treatment modality. Occult metastatic spread of cancer cells from primary tumor to surrounding tissues occurs in more than $50 \%$ of lung cancer patients at the time of diagnosis of lung cancer, and it has a very negative effect on patient's prognosis. The prognosis of patients who undergo curative surgical resection in the early stage of lung cancer is improved with an adjuvant chemotherapy. Thus, identifying biomarkers for early detection of lung cancer and personalized medicine is very important for prolonging the lifespan of lung cancer patients. However, sensitive and specific biomarkers for detecting lung cancer are currently not available in clinical setting.

Mammalian SLIT1, SLIT2 and SLIT3 genes are located on human chromosome 10q24.1, 4p15.31, and 5q34, respectively. SLITs (SLIT1-3) are highly conserved secreted glycoproteins in vertebrates and mediate biologic effects on cells by interacting with transmembrane receptor ROBOs (ROBO1-4) in many physiological and pathological processes [2]. The SLITROBO pathway is crucial for muscle cell formation, neuronal axon guidance, cell migration, cell proliferation, inflammatory cell chemotaxis and angiogenesis. SLITs and ROBOs are highly expressed in many types of cancer, and the SLIT/ROBO signaling pathway has a positive effect on tumor growth, tumor cell migration, and tumor metastasis [3]. Compared with studies reporting an oncogenic role of SLIT proteins, recent studies have also indicated that they can function as tumor suppressors by inhibiting cancer cell proliferation, cancer progression, and metastasis. The inactivation of SLIT proteins has been reported in several types of cancer, such as breast cancer [4-6], colorectal cancer [7,8], gastric cancer $[9,10]$, hepatocellular carcinoma [11,12], lung cancer [13-16] and thyroid cancer [17].

Hypermethylation of $\mathrm{CpGs}$ located at the promoter regions of tumor suppressor genes is now well established as an important mechanism of epigenetic inactivation. Hypermethylation of SLIT1 gene has been found in colon cancer [18,19] and glioma [20]. However, it has not been reported in lung cancer yet, although hypermethylation of SLIT2 [21-24] and SLIT3 [20,22,24] has been reported in lung cancer.

To understand their feasibility as biomarkers for early detection and survival prediction of non-small cell lung cancer (NSCLC), we analyzed methylation statuses of SLIT1, SLIT2 and SLIT3 genes using the Infinium HumanMethylation450 BeadChip in 136 bronchial washings, 6 bronchial biopsies, 12 sputums, and 42 lung tumor and matched normal tissues. SLIT2 methylation was analyzed using pyrosequencing in 133 plasma samples.

\section{Materials and Methods}

\subsection{Study Population and Samples}

A total of 208 NSCLC patients and 121 cancer-free patients who were admitted for diagnosis of suspected lung cancer or for curative surgical resection of lung cancer or who visited for a regular health check-up at Samsung Medical Center in Seoul, Korea between August 1994 and August 2016 participated in this study. The cancer-free patients were recruited from healthy individuals that have not been diagnosed with any cancer till August 2020 or from patients with benign lung diseases such as pneumonia, bronchiolitis, tuberculosis, or actinomycosis. Localized organizing pneumonia and hamartoma were excluded from the study because methylation profiling of both diseases is not well known, which could lead to misclassification. Information related to tissue, bronchial washing, sputum, and bronchial biopsy samples used in this study was described previously [25]. Plasma samples collected after July 2005 were used for analysis of SLIT2 methylation. To avoid misclassification of case and control, patients with other cancers found during the follow-up for at least 5 years after lung cancer surgery or bronchoscopy or a regular health check-up were excluded from this study. Venous blood was collected into $10 \mathrm{~mL}$ Vacutainer ${ }^{\circledR} \mathrm{K}_{3}$ EDTA tubes (BD Medical, Franklin Lakes, NJ, USA), kept in a refrigerator and then centrifuged at $1700 \times g$ for $10 \mathrm{~min}$ at $4{ }^{\circ} \mathrm{C}$. The time between sample collection and plasma separation was a minimum of one hour and a maximum of eight hours (Figure S1). Plasma was stored at $-80^{\circ} \mathrm{C}$ until use. Follow-up after curative resection was conducted by 
a lung cancer specialized nurse as described previously [26]. This study was approved by the Institutional Review Board of the Samsung Medical Center (IRB\#: 2010-07-204). It was conducted in accordance with the Declaration of Helsinki. Written informed consent for the use of pathological specimens was obtained from all participants prior to the procedure. Tumor/node/metastasis (TNM) system provided by The American Joint Committee on Cancer (AJCC) [27] was used for pathologic staging of NSCLC.

\subsection{Analysis of SLITs Methylation}

We have previously analyzed CpGs methylation in 6 bronchial biopsies, 12 sputums, 42 surgically resected tumor and matched normal tissues and 136 bronchial washings obtained from 76 NSCLC patients and 60 cancer-free patients using the Infinium HumanMethylation450 BeadChip [25]. We used the reported data for analyzing aberrant methylation of SLIT1, SLIT2, and SLIT3 genes in tumor tissues and bronchial aspirates. Data preprocessing such as batch effect adjustment, background correction, probe filtering and adjustment of performance difference between type I and II probes was performed using the wateRmelon $\mathrm{R}$ software package (version 3.1.1). Methylation level ( $\beta$-value) for each cytosine in a $\mathrm{CpG}$ dinucleotide was estimated as the ratio of fluorescent signal from methylated cytosines to the sum of methylated and unmethylated cytosines. CpGs were defined as hypermethylated when $\beta$-value was greater than or equal to 0.3 under consideration of background signal.

\subsection{Feature Selection and Model Building for Lung Cancer Prediction}

To select tumor-specific CpGs for lung cancer prediction and model building among differentially methylated CpGs, we randomly divided normal and tumor tissues into training and test datasets at a ratio of 7:3. Supervised machine learning algorithms were applied for feature selection and model building using RapidMiner Studio (version 8.2) in the training dataset. Age-related CpGs and any significantly correlated CpGs in normal or tumor tissues were removed from the model building. The performance of each prediction model was evaluated with test datasets using a receiver operating characteristic (ROC) curve, which was plotted using the statistical software package MedCalc (version 19.0.5).

\subsection{Analysis of SLIT2 Methylation in Plasma}

Methylation levels of cg13281139 locus at the promoter region of SLIT2 gene were evaluated by pyrosequencing using a PyroMark Q24 ID System (Qiagen, Hilden, Germany) according to the manufacturer's protocol. DNA was extracted from $2 \mathrm{~mL}$ of plasma using the MagMax Cell-Free DNA Isolation Kit (Thermo Fisher Scientific, Waltham, MA, USA) according to the manufacturer's protocol. The quantity and size of the extracted DNA were measured using an Agilent High Sensitivity DNA Kit (Santa Clara, CA, USA, Part no. 50674626). Contaminated genomic DNA was removed using $2 \%$ isopropanol. Approximately $20 \mathrm{ng}$ of DNA was treated with bisulfite using an EZ DNA Methylation Gold kit (Zymo Research, Irvine, CA, USA) according to the manufactures' instructions. Bisulfite-treated DNA was then eluted in $10 \mu \mathrm{L}$ elution buffer. Primers for pyrosequencing were designed using the PyroMark Assay Design 2.0 software (Qiagen), and primer sequences are listed in Table S1. SLIT2 was amplified uisng a PyroMark PCR kit (cat. No 978703; Qiagen) according to the manufacturer's protocol. Briefly, PCR was performed on a Gene PCR System 9700 (Applied Biosystems, Foster City, CA, USA). PCR was carried out in a final volume of $25 \mu \mathrm{L}$ comprising $12.5 \mu \mathrm{L}$ of PyroMark PCR Master Mix $(2 \times), 1 \mu \mathrm{L}$ of modified DNA, $0.5 \mu \mathrm{L}$ each of forward and biotinylated reverse primers ( $0.2 \mu \mathrm{M}$ final concentration), $2.5 \mu \mathrm{L}$ of CoralLoad buffer $(10 \times)$, and $8 \mu \mathrm{L}$ of RNase-free water. PCR was started with 1 cycle of $95{ }^{\circ} \mathrm{C}$ for $15 \mathrm{~min}$, followed by 45 cycles of $95^{\circ} \mathrm{C}$ for $15 \mathrm{~s}, 60{ }^{\circ} \mathrm{C}$ for $20 \mathrm{~s}, 72{ }^{\circ} \mathrm{C}$ for $20 \mathrm{~s}$, and 1 cycle of $72{ }^{\circ} \mathrm{C}$ for $10 \mathrm{~min}$. PCR products were sequenced on a PyroMark Q24 (Qiagen) using PyroMark Gold Q24 reagents (Qiagen) according to the manufactures' instructions. To check the accuracy of pyrosequencing, we made $0 \%, 25 \%, 50 \%$ and $100 \%$ methylated samples by mixing methylated (Chemicon, Millipore, Billerica, MA, USA) and 
unmethylated control DNA (Qiagen) and included them in all experiments. Results were analyzed using the PyroMark Q24 2.0.6 software (Qiagen).

\subsection{Statistical Analysis}

For univariate analysis, the Wilcoxon rank sum test (or $t$-test) and the Fisher's exact test (or Chi-square test) were used for continuous and categorical variables, respectively. The correlation of methylation levels of selected CpGs was assessed using Spearman's rank correlation coefficient. The effect of SLITs hypermethylation on overall survival or recurrence-free survival (RFS) in lung cancer patients was estimated using the KaplanMeier survival curve, and the difference between two survival curves was evaluated by the log-rank test. The hazard ratio of SLITs hypermethylation for survival was estimated using Cox proportional hazards regression model after adjusting for potential confounding factors. All statistical analyses were conducted using R software (version 3.6.1).

\section{Results}

\subsection{Aberrant Methylation of SLIT Genes in Primary Non-Small Cell Lung Cancer}

To identify differentially methylated CpGs at the promoter regions of SLIT genes, we analyzed methylation statuses of SLIT1, SLIT2 and SLIT3 genes in tumor and matched normal tissues from 42 NSCLC patients previously reported [25]. Methylation levels of CpGs in tumor tissues were negatively skewed and did not follow a normal distribution (Shapiro-Wilk test, $p<0.05$ ). Thus, the Wilcoxon rank sum test was applied to assess the statistical significance. Five CpGs (Figure 1A) with a $p$-value less than or equal to $1.03 \times 10^{-7}$ (Bonferroni-corrected threshold) were identified from the $450 \mathrm{~K}$ array. Information on these five CpGs is described in Table S2. Average methylation levels of these five CpGs in tumor tissues were 0.67-0.84. A linear relationship between methylation levels of these CpGs was analyzed in 42 tumor tissues (Figure 1B). Two CpGs (cg13261826 and cg14226472) in SLIT1 gene were significantly correlated to each other, and a statistically significant correlation was also found between CpGs at the promoter region of SLIT1 (cg13261826 or cg14226472) and SLIT3 (cg01163016 or cg26119620). However, a CpG (cg03260566) in SLIT2 was not correlated to any CpGs in SLIT1 or SLIT3. The association between hypermethylation of $\mathrm{CpGs}$ and clinicopathological variables was analyzed. Hypermethylation of the five CpGs was not associated with lymphatic invasion of tumor cells (Wilcoxon rank sum test; Figure 1C). Methylation of cg01663016 (SLIT3) was associated with patient age $(r=0.40, p=0.009$; Figure 1D). Hypermethylation of three CpGs (cg13261825, cg03260566 and cg01663016) in SLIT1, SLIT2 and SLIT3 genes was found at a higher prevalence in adenocarcinoma than in squamous cell carcinoma, although the difference was not statistically significant (Figure 1E). Methylation levels of the five CpGs were not associated with pack-years, tumor size, pathologic stage, vascular invasion or neural invasion Relationship between hypermethylation of cg03260566 in SLIT2 and the clinical parameters was shown in Table S3. 
A

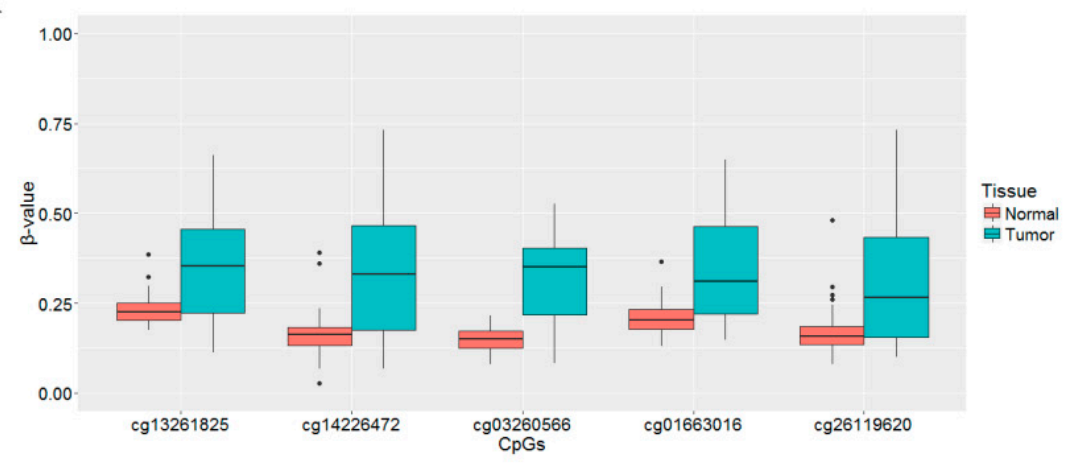

C

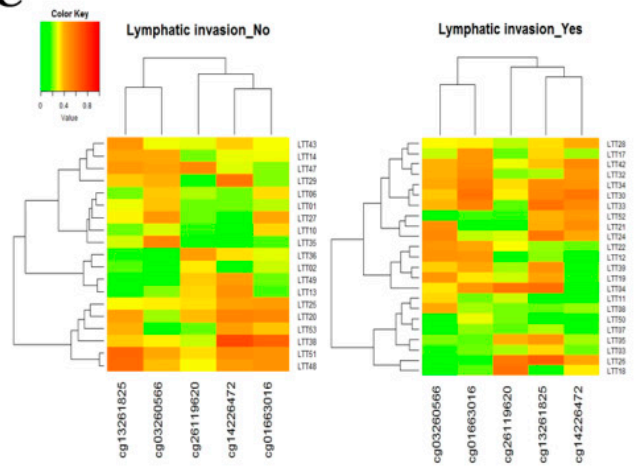

D

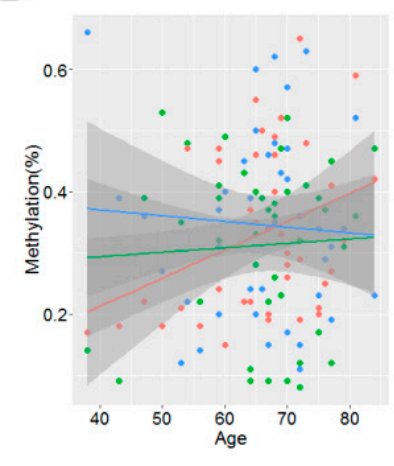

B

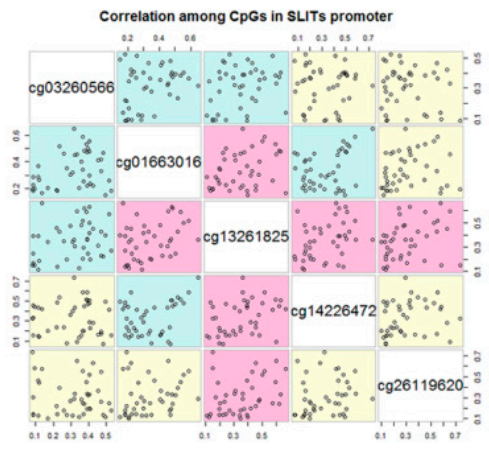

$\mathbf{E}$

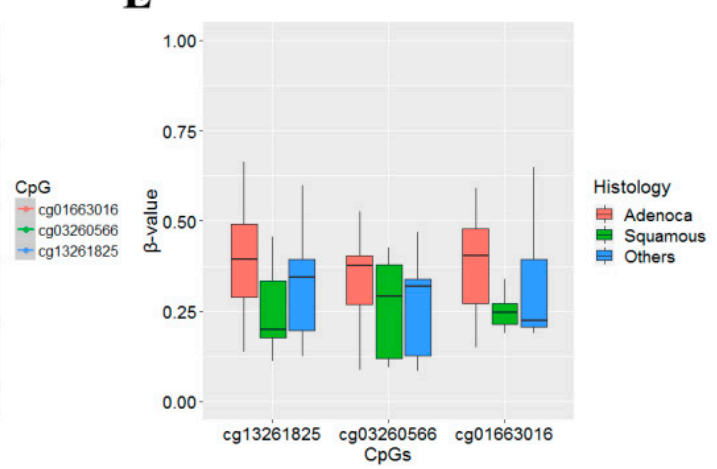

Figure 1. Relationship between aberrant methylation of SLIT genes and clinicopathological features in 42 patients with NSCLC. (A) Methylation levels of five CpGs at the CpG island regions of SLIT1, SLIT2, and SLIT3 genes were compared between tumor and matched normal tissues from 42 NSCLC patients. Y-axis indicates $\beta$-value. (B) Correlation among methylation levels of five CpGs was analyzed for 42 tumor tissues using Spearman's correlation coefficients. Magenta color indicates $p<0.05$. (C-E) Methylation levels of CpGs at SLIT1, SLIT2 and SLIT3 genes were compared according to lymphatic invasion (C), age (D), and histologic subtypes (E).

\subsection{Aberrant Methylation of SLIT Genes in Bronchial Washing, Biopsy, and Sputum Samples}

Methylation levels of three CpGs (cg13261825, cg03260566, and cg01663016) were analyzed in 136 bronchial washings (76 NSCLC patients and 60 cancer-free patients), 12 sputum samples and six bronchial biopsy specimens from NSCLC patients to test if those specimens could be used as surrogate samples for assessing aberrant methylation of SLIT genes observed in lung tumor tissues. Methylation levels of these three $\mathrm{CpGs}$ (Figure 2A-C) in bronchial biopsy, bronchial washing and sputum specimens were significantly lower than those in tumor tissues shown in Figure 1A, but similar to those in normal tissues. In addition, methylation levels of these CpGs in bronchial biopsy samples from NSCLC patients were not significantly different from those in 6 paired bronchial washing samples ( $p>0.05$, Wilcoxon signed-rank test; Figure 2D-F). Based on these observations, sputum, bronchial washing and bronchial biopsy specimens might not be feasible as surrogates for analyzing aberrant methylation of SLIT genes in lung tumor tissues. 
A

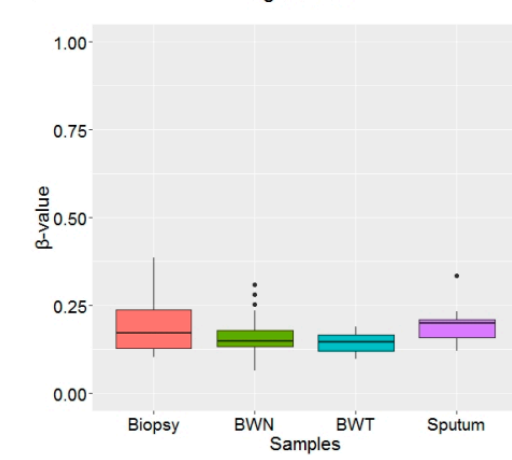

D

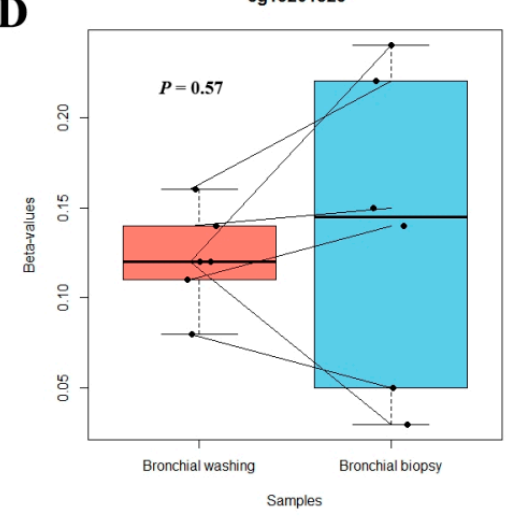

B

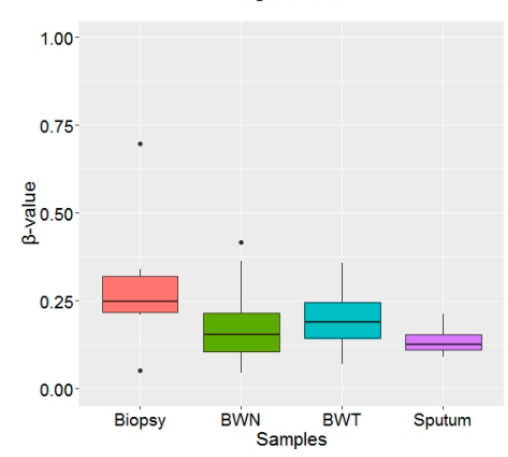

$\mathbf{E}$

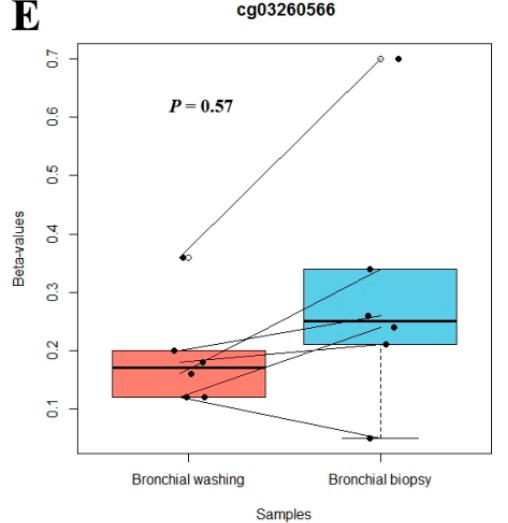

C

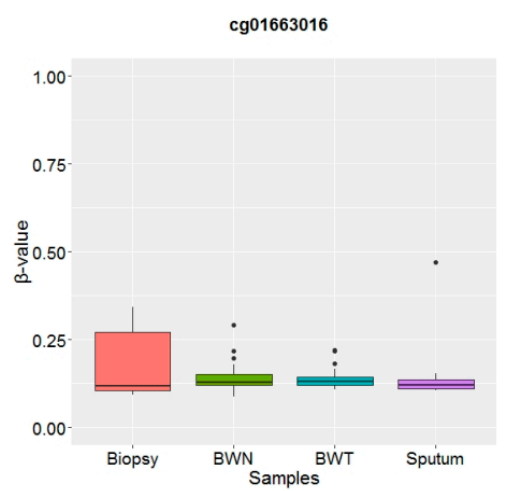

$\mathbf{F}$

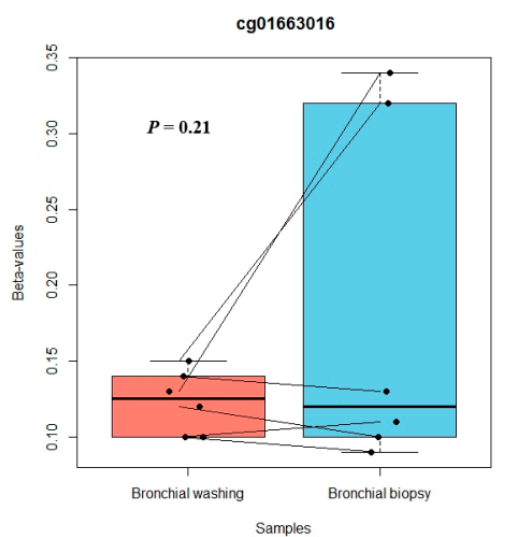

Figure 2. Aberrant methylation of SLIT genes in bronchial washing, biopsy, and sputum samples. (A-C) Methylation levels of three CpGs ((A) cg13261825, (B) cg03260566 and (C) cg01663016) were compared in six bronchial biopsy and 12 sputum samples from NSCLC patients and in bronchial washing samples from 60 cancer-free patients (BWN) and 76 NSCLC patients (BWT). (D-F) Methylation levels of these three CpGs were also analyzed between paired bronchial washing and bronchial biopsy specimens from six NSCLC patients. $p$-values are based on Wilcoxon signed rank test. Y-axis indicates $\beta$-value.

\subsection{Prediction of NSCLC Using Methylation Levels of SLIT Genes in Lung Tumor Tissues}

To select features and develop a model for predicting NSCLC, we divided 42 lung tumor and matched normal tissues into a training dataset and a test dataset at a ratio of 7 to 3, respectively. The training dataset $(N=59)$ was used for feature selection and model building, and the performance of each model was tested using the test dataset $(N=25)$. Supervised machine learning algorithms such as Naive Bayes (NB), logistic regression, k-nearest neighbor (kNN), support vector machine (SVM), artificial neural network (ANN) and random forest were applied for selecting features and building a model. Correlated CpGs were not included in these models at the same time. Among applied algorithms, a logistic regression model based on cg03260566 in a test dataset $(N=25)$ showed the best performance (sensitivity of $84.2 \%$ and specificity of $79.8 \%$ ) in predicting NSCLC, with the area under the curve (AUC) of 0.76 (95\% CI =0.71-0.92, $p<0.0001$; Figure 3A). We further evaluated the prediction performance of the same model in TCGA lung cancer data (824 tumor and 74 normal tissues) to understand whether SLITs methylation could be applied to other races. Methylation levels of four CpGs in the Cancer Genome Atlas (TCGA) data were similar to those in our data (Figure S2). The TCGA lung cancer data were also divided into a training dataset $(N=630)$ and a test dataset $(N=268)$ for building a model. A logistic regression model based on cg03260566 in the test dataset predicted NSCLC with a sensitivity of $87.2 \%$ and a specificity of $78.4 \%(\mathrm{AUC}=0.83 ; 95 \% \mathrm{CI}=0.73-0.92, p<0.0001$; Figure $3 \mathrm{~A}$ ). The prediction certainty of NSCLC was found to be high in our data and the TCGA test dataset (Figure 3B). 
A

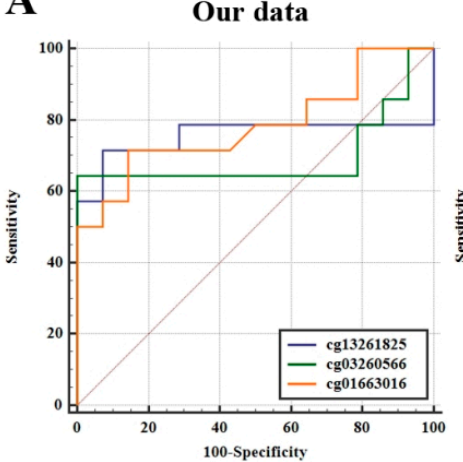

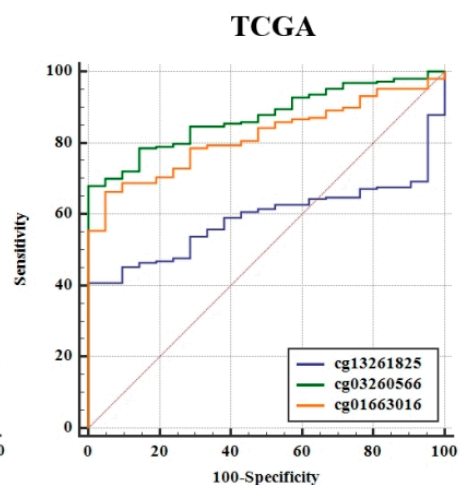

B

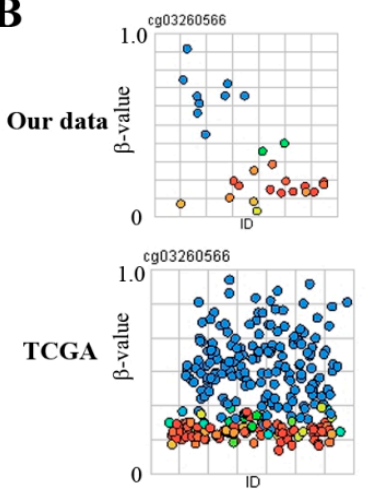

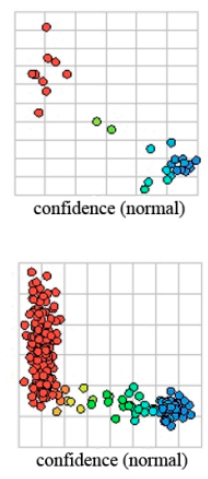

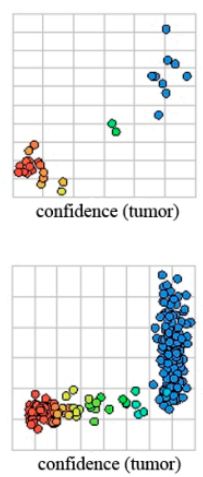

Figure 3. Evaluation of prediction performance of a model in our data and TCGA lung cancer data. (A) Prediction performance of a logistic regression model based on three CpGs was evaluated in our data (left) and TCGA (right) test dataset. ROC curves were plotted using the MedCalc software. (B) Prediction certainty of a logistic regression model based on cg03260566 was estimated in a test dataset. Y-axis indicates $\beta$-value. "ID" in the left panel represents identification number of test samples. In the middle and right panels, the $\mathrm{X}$-axis indicates confidence (normal or tumor) that predicts samples as normal (middle panel) or tumor (right panel) for each $\beta$-value on the Y-axis. The sky blue and red orange circles indicate high and low confidence, respectively.

\subsection{Aberrant Methylation of SLIT2 in Plasma Cell-Free DNA of Patients with NSCLC}

Clinicopathological characteristics of 72 NSCLC patients and 61 cancer-free patients who donated blood samples are described in Table S4. Genomic DNA (Figure 4A) frequently found in plasma cell-free DNA was removed using $2 \%$ isopropanol. The amount of plasma cell-free DNA after isopropanol was very small, and therefore only SLIT2 methylation was analyzed using pyrosequencing. Pyrosequencing of cg03260566 showing a high performance in predicting NSCLC in tissue samples failed due to an unequal amplification of methylated and unmethylated alleles in PCR reaction. Instead, methylation levels of cg13281139 located approximately $1200 \mathrm{bp}$ upstream from cg03260566 were analyzed. Average methylation levels of cg13281139 were 0.16 and 0.08 in plasmas from 72 NSCLC patients and 61 cancer-free patients, respectively, and the difference was statistically significant irrespective of gender ( $p=0.001$, Wilcoxon rank sum test; Figure $4 \mathrm{~B}$ ). To build a model for lung cancer prediction using methylation levels of cg13281139 in plasma cell-free DNA, the data were divided into a training dataset $(70 \%)$ and a test dataset $(30 \%)$. The sensitivity and specificity of a logistic regression model based on cg13281139 in the test dataset $(N=40)$ were $73.7 \%$ and $61.9 \%$, respectively (Figure $4 \mathrm{C}$ ). This finding suggests that SLIT2 may be a valuable biomarker to detect NSCLC in plasma cell-free DNA.

\subsection{SLIT2 Hypermethylation in Plasma Cell-Free DNA Is Associated with Poor Recurrence-Free Survival of NSCLC Patients}

Clinicopathological significance of SLIT2 hypermethylation was analyzed in plasma cell-free DNA from 72 NSCLC patients. SLIT2 was defined as hypermethylated when $\beta$-value was greater than or equal to 0.15 . SLIT2 hypermethylation was found in 24 (33\%) of 72 NSCLCs. SLIT2 hypermethylation was not significantly different according to gender (Figure 5A), histology (Figure 5B), or pathologic stage (Figure 5C). In addition, SLIT2 hypermethylation was not associated with age, smoking status, tumor size, lymphatic invasion, vascular invasion or neural invasion. However, SLIT2 hypermethylation was significantly associated with tumor recurrence (Figure 5D). The median follow-up period of patients was 5.2 years. Patients with SLIT2 hypermethylation showed poor recurrence-free survival (RFS) compared to those without (Figure 5E). Five-year recurrence-free survival rates of patients with and without SLIT2 hypermethylation were $44 \%$ and $80 \%$, respectively. Cox proportional hazards analysis showed that RFS of patients with SLIT2 hypermethylation 
was 2.19 times $(95 \% \mathrm{CI}=1.21-4.36 ; p=0.01)$ poorer than in those without after adjusting for age and pathologic stage (Table 1$)$.

$\mathbf{A}$

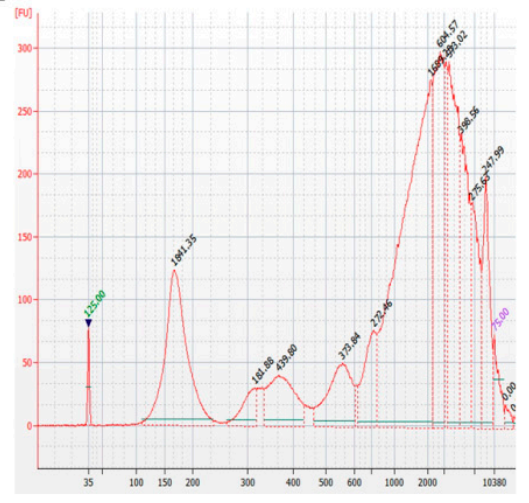

B

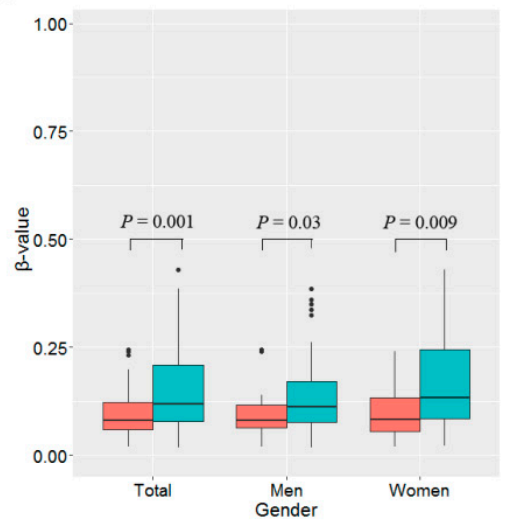

C

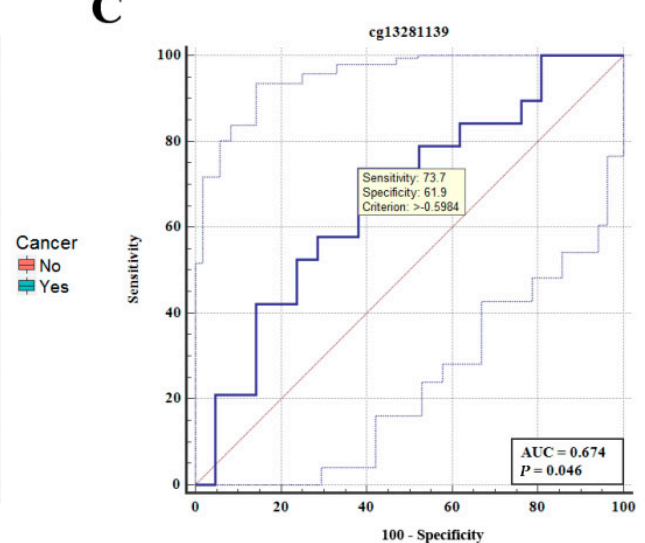

Figure 4. Analysis of SLIT2 methylation in plasma cell-free DNA of NSCLC patients. (A) Cell-free DNA extracted from plasma was analyzed using an Agilent DNA high sensitivity Kit on an Agilent Bioanalyzer 2100 instrument. Electropherogram shows representative sample with contaminated genomic DNA fragments longer than 400 bp. (B) Methylation levels of SLIT2 (cg13281139) in plasma cell-free DNA were compared between 72 NSCLC patients and 61 cancer-free patients according to gender. $p$-values were based on Wilcoxon rank sum test. (C) True positive (sensitivity) and false positive (1-specificity) rates of a logistic regression model based on cg13281139 in plasma cell-free DNA were evaluated with a test dataset $(N=40)$. ROC curve with $95 \%$ confidence interval was plotted using the MedCalc software (version 19.0.5).

A

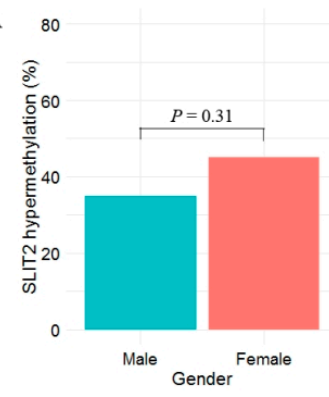

C

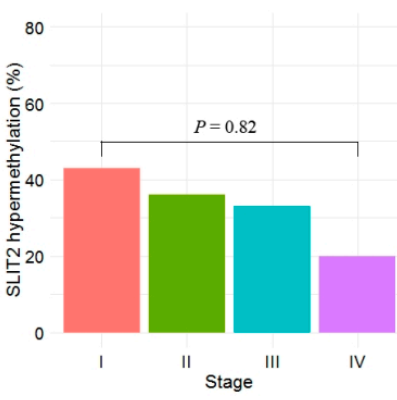

B

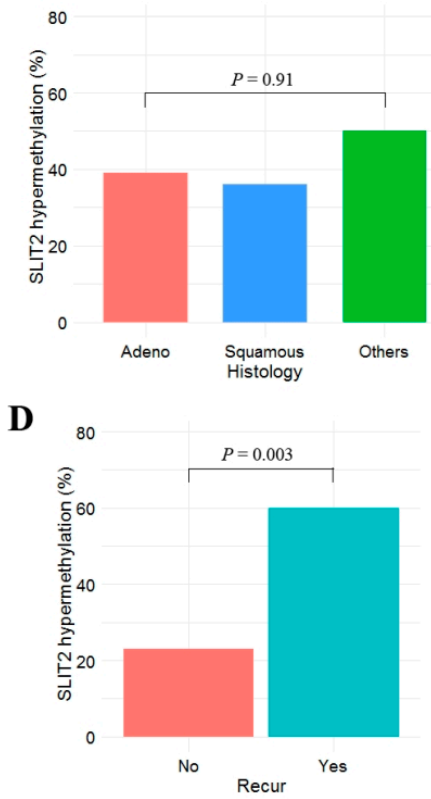

$\mathbf{E}$

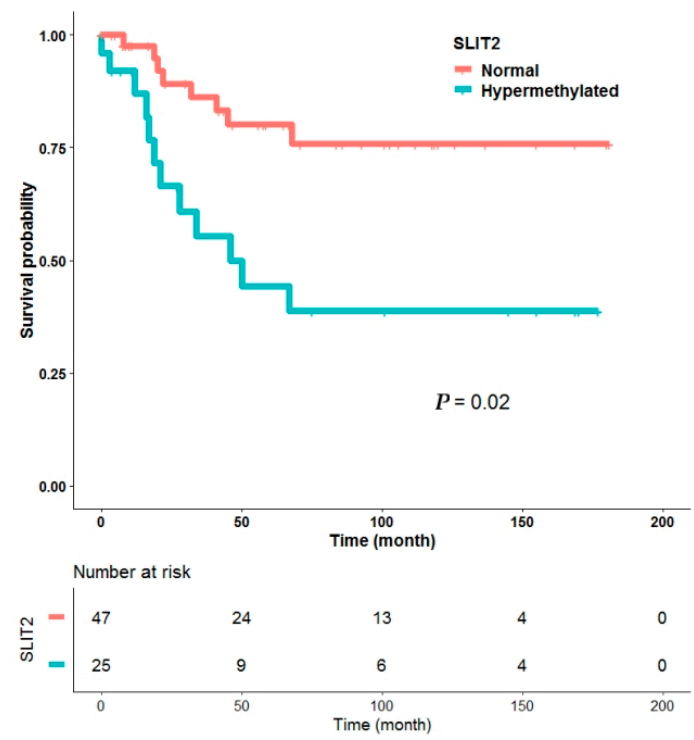

Figure 5. Negative effect of SLIT2 hypermethylation in plasma cell-free DNA on recurrence-free survival of NSCLC patients. (A-D) Methylation levels of SLIT2 in plasma cell-free DNA from 72 NSCLC patients were compared according to gender (A), histology (B), pathologic stage (C), and recurrence (D). "Adeno" and "Squamous" indicate "adenocarcinoma" and "squamous cell carcinoma", respectively. $p$-values were based on Fisher's exact test or Chi-square test. (E) The relationship between methylation level of SLIT2 in plasma cell-free DNA and recurrence-free survival was analyzed using Kaplan-Meier survival estimates, and $p$-value was calculated using the logrank test. 
Table 1. Cox proportional hazards analysis of survival according to SLIT2 hypermethylation.

\begin{tabular}{ccccc}
\hline Survival & SLIT2 Hypermethylation & HR & 95\% CI & $p$-Value \\
\hline \multirow{2}{*}{ Overall survival } & No & 1.00 & - & - \\
Recurrence-free & Yes & 1.84 & $0.76-5.11$ & 0.38 \\
survival & No & 1.00 & - & - \\
\hline
\end{tabular}

Abbreviations: HR, hazard ratio; CI, confidence interval. ${ }^{1}$ Adjusted for age and pathologic stage.

\section{Discussion}

SLIT proteins interact with ROBO receptors, thus playing important roles in cell migration, cell growth, and angiogenesis. Hypermethylation of SLIT genes has been reported in diverse types of cancer, including lung cancer. To understand whether hypermethylation of SLIT genes can be used as a biomarker for early detection and prognosis prediction of NSCLC, we analyzed methylation statuses of SLIT genes in lung cancer tissues, bronchial washing, biopsy and plasma samples. SLIT1 expression is specific to brain and the nervous system [20] and its hypermethylation has not been reported in lung cancer. However, hypermethylation of SLIT1 in this study was found in $29(69.0 \%)$ of 42 NSCLC tissues. Hypermethylation of SLIT2 and SLIT3 occurred in $29(69.0 \%)$ and $23(54.8 \%)$ of 42 NSCLCs, respectively, consistent with previous findings reporting that the frequency of SLIT3 methylation in lung cancer was less than that of SLIT2 $(20,22)$. This suggests that an alternative mechanism other than hypermethylation is responsible for the downregulation of SLIT3. Methylation levels of SLITS were compared with those in the TAGA lung cancer data. Similar results were obtained between our data and TCGA lung cancer data (Figure S2). Although the number of normal tissues $(N=74)$ in the TCGA lung cancer data was only about one-tenth of that of tumor tissues $(N=824)$ and the incidence of lung cancer between Koreans and Americans was not exactly the same, the present study suggests that the clinicopatholgocial significance of SLIT genes in the present study might be applicable to other races.

Cytologic examination of sputum turned out to be ineffective in enhancing lung cancer detection and reducing lung cancer mortality. To improve their poor efficiency, a number of groups have analyzed methylation changes of multiple tumor suppressor genes in exfoliated bronchial epithelial cells shed from the respiratory tract in addition to sputum. In this study, methylation levels of SLIT genes in lung tumor tissues were significantly higher from those in sputum, bronchial washing, and bronchial biopsy samples. In addition, any CpGs in SLIT genes on the Illumina Human Methylation $450 \mathrm{~K}$ BeadChip did not pass through the Bonferroni corrected significance threshold in exfoliated bronchial epithelial cells obtained through bronchial washing from 76 NSCLC patients and 60 cancer-free patients. Given the likelihood of detecting aberrant methylation in bronchial washing samples depended on tumor location and given that bronchial washing specimens were not good materials for centrally located tumors such as squamous cell carcinoma, we also analyzed methylation changes in bronchial washing samples from squamous cell carcinoma and adenocarcinoma patients separately However, SLITs methylation showed no difference between peripherally located adenocarcinomas and cancer-free patients and paired bronchial washing and biopsy samples showed no significant difference in methylation levels of SLIT genes either. Based on these results, sputum, bronchial washing and biopsy specimens might not be useful surrogates for analyzing methylation statuses of SLIT genes in lung cancer.

Circulating cell-free DNA is a mixture of DNA derived from tumor and normal tissues and is known to contain tumor-associated aberrant methylation in various types of cancer. Analysis of methylation in plasma cell-free DNA through liquid biopsy is considered to have potential for early detection of cancer. Although many epigenetic biomarkers have been discovered for that purpose, it is still challenging to identify biomarkers with clinical accuracy in plasma cell-free DNA. This might be due to the technical difficulty in analyzing highly fragmented cell-free DNA. Among many methods for analyzing CpG methylation 
in plasma cell-free DNA, pyrosequencing was used in this study. However, we failed to amplify a DNA segment including cg03260566 in plasma cell-free DNA from NSCLC patients. We searched other CpGs around cg03260566 and ultimately analyzed cg13281139, which was approximately $1200 \mathrm{bp}$ away from cg03260566. In tumor tissues, cg13281139 was found to be less frequently hypermethylated than cg03260566 (Figure S3), and methylation status of cg13281139 did not show any relationship to clinical parameters (Table S3). A significant drawback in this study was the inability to obtain samples from patients' premalignant disease such as dysplasia and adenoma to analyze its potential as a biomarker for early detection of NSCLC. Therefore, we have compared the methylation levels of cg13281139 in bronchial washing from 60 healthy individuals, matched normal tissues from 42 NSCLC patients, and tumor tissues from stage I-III NSCLC patients (Figure S4). A significant increase in the methylation levels of cg13281139 in stage I compared to normal bronchial washing samples and matched normal tissues suggests the possibility as a biomarker for early detection of NSCLC, but further studies are needed in a large cohort including premalignant lesions. In addition, the sensitivity and specificity of NSCLC prediction using cg13281139 in plasma cell-free DNA were not high (Figure 4C). Failure of methylation analysis in plasma cell-free DNA usually results from primer design failure, uneven amplification of methylated and unmethylated alleles, or amplification failure of fragmented cell-free DNA. These were eventually caused by a lack of knowledge about fragments of cell-free DNA. Internucleosomal linker DNA is preferentially digested by endonuclease during an apoptotic DNA fragmentation [28], and cutting sites in linker DNA are also affected by the density of CpG methylation [29]. To analyze more reliable CpGs in plasma cell-free DNA, it is critical to understand the nucleosomal positioning pattern and ending patterns from plasma cell-free DNA.

In this study, mRNA levels of SLITs and ROBOs were analyzed using data previously obtained from HumanHT-12 expression BeadChips (Illumina, San Diego, CA, USA) in 42 surgically resected tumor and matched normal tissues [30]. mRNA levels of SLIT2, $\mathrm{ROBO} 2, \mathrm{ROBO} 3, \mathrm{ROBO} 4$ were significantly downregulated in tumor tissues compared to normal tissues (Table S5). The association between SLIT2 downregulation and patient's prognosis has been reported in different types of cancer. For example, low expression of SLIT2 is correlated with an upward trend of pathological stage in NSCLC, poor overall and disease-free survival [13]. Knockdown of SLIT2 increases migration of esophageal squamous cells, and low expression of SLIT2 protein is correlated with poor overall survival and disease-free survival in esophageal squamous cell carcinoma [31]. Expressions of SLIT2 and ROBO1 are downregulated in ductal carcinoma in situ and invasive breast cancer tissues, and such downregulation is associated with poor prognosis and brain metastasis of breast cancer [32]. Survival time for brain glioma patients with decreased SLIT2 mRNA expression is shorter than for that for those with a normal expression of SLIT2 [33]. Reduced mRNA expression of SLIT2 is associated with advanced clinical stage and worse overall survival in diffuse large B cell lymphoma [34]. Papillary thyroid cancers patients with negative SLIT2 expression have significantly increased risk of recurrence and metastasis [17]. SLIT2 mRNA levels in this study showed a negative trend with methylation levels of cg13281139, but were not statistically significant (Figure S5). Nonetheless, the present study suggests that SLIT2 methylation status in plasma cell-free DNA might be helpful for tracking the recurrence of NSCLC patients. Further studies are needed in a large cohort.

Emerging evidence suggest that SLITs and ROBOs as tumor suppressors act by inhibiting cancer cell proliferation, angiogenesis, invasion, and migration in different types of cancer. Mechanisms of action of SLITs on lung cancer have also been reported by several groups. SLIT2 is frequently inactivated in lung cancer, and knockdown of SLIT2 decreases the interaction between beta-catenin and E-cadherin/SNAI1 in lung cancer cells, resulting in increased cell migration and reduced cell adhesion [13]. SLIT2 suppresses the migration and invasion of lung cancer cells by regulating RhoA activity [14]. The expression of SLIT3 is downregulated in lung tumor tissues and silencing of SLIT3 in lung cancer cells 
induces epithelial-mesenchymal transition by downregulating E-cadherin and enhancing MMP2 and MMP9 expression [35]. In the present study, relationships of SLITs methylation with tumor size, pathologic stage, vascular, neural invasion and lymphatic invasion were analyzed, showing no significant relationship. These inconsistent results between in vitro and in vivo studies might be due to the complexity of SLIT/ROBO signaling pathway in tumor proliferation, invasion and metastasis. In this study, the relationship between ROBO expression levels and methylation levels of cg13281139 was analyzed to understand potential cellular mechanisms underlying the negative effect of cg13281139 methylation on RFS. However, no relationship was found between them (Figure S6). Thus, the role of SLITs in clinical lung cancer samples needs to be analyzed by taking into account the expression and/or methylation status of ROBOs and other proteins in the downstream of SLITs.

For analysis of cell-free DNA, it is of the utmost importance to optimize pre-analytical conditions that can affect sample integrity. Several groups reported guidelines for preanalytical conditions for the analysis of cell-free DNA [36,37]. The interval between blood collection and plasma separation is known to be a factor that can affect the integrity of cell-free DNA. In this study, all plasmas were separated within eight hours of blood collection (Figure S1), and the significant contamination of genomic DNA (Figure 4A) was mostly found in samples whose plasma separation was performed after six hours of blood collection. Accordingly, plasma needs to be isolated as early as possible although blood is stored at $4{ }^{\circ} \mathrm{C}$ after collection.

This study was limited by several factors. First, the present study was a retrospective case-control study, which could give rise to a highly biased estimate in population prevalence of NSCLC. Accordingly, prediction performance of a model needs to be validated using a cohort study in different populations. Second, in addition to a small number of total plasma samples, the statistical power was insufficient because the number of normal plasma samples was small compared to that for lung cancer patients. Third, methylation levels of SLIT1 and SLIT3 in plasma cell-free DNA were not analyzed due to insufficient amounts of cell-free DNA. SLIT2 methylation levels were not correlated with SLIT1 or SLIT3 methylation. Thus, the addition of SLIT1 or SLIT3 methylation level to the present prediction model might improve the prediction performance of NSCLC. Fourth, the mechanism of action of SLIT2 in inhibiting recurrence of NSCLC was not fully elucidated. Fifth, there was significant genomic DNA contamination in plasma (Figure 4A) due to the long interval between blood collection and plasma separation. The genomic DNA was properly removed using 2\% isopropanol, but loss of cell-free DNA was also significant (Figure S7). Sixth, there was a possibility that degradation of genomic DNA from lymphocytes results in small fragments that may increase the amount of plasma cell-free DNA. However, we could not separate small DNA fragments of lymphocytes from cell-free DNA technically.

In conclusion, the present study suggests that aberrant methylation of SLIT2 gene in plasma cell-free DNA might be a potential biomarker for detecting NSCLC and predicting recurrence-free survival. Further research is needed with larger sample size to confirm results.

Supplementary Materials: The following supporting information can be downloaded at: https:/ / www.mdpi.com/article/10.3390/cancers14020296/s1. Figure S1, Distribution of plasma separation time after blood collection; Figure S2: Aberrant methylation of SLIT genes in TCGA lung cancer cohort; Figure S3: Degree of methylation of cg03250566 and cg13281139 in NSCLC; Figure S4: Methylation levels of cg03250566 and cg13281139 according to pathologic stage; Figure S5: Correlation between cg13281139 methylation and SLIT2 mRNA levels; Figure S6: Relationship between cg13281139 methylation and ROBOs expression; Figure S7: Electropherogram after clearance of genomic DNA with $2 \%$ isopropanol. Table S1, Primer sequences for pyrosequencing; Table S2: CpGs information; Table S3: Relationship between SLIT2 hypermethylation and clinicopathological variables $(N=42)$; Table S4: Clinicopathological characteristics; Table S5: mRNA fold change of SLIT2 and ROBOs in 42 NSCLCs. 


\begin{abstract}
Author Contributions: Y.K. and D.-H.K. designed the overall study, analyzed the data, and drafted the manuscript. Y.K., B.B.L. and D.K. contributed to cell-free DNA preparation, genome-wide methylation analysis and pyrosequencing. Bronchoscopy was performed by S.-W.U. and pathologic diagnosis was made by J.H. All participants' curative resection of non-small cell lung cancer was conducted by Y.M.S. All authors have read and agreed to the published version of the manuscript.
\end{abstract}

Funding: This work was supported by a grant (2019R1F1A1057654) from the Basic Science Research Program through the National Research Foundation (NRF) funded by the Ministry of Education, Republic of Korea.

Institutional Review Board Statement: The study was conducted in accordance with the Declaration of Helsinki, and approved by the Institutional Review Board at the Samsung Medical Center IRB \#: 2010-07-204.

Informed Consent Statement: Informed consent was obtained from all subjects involved in the study.

Data Availability Statement: The data presented in this study are available in the article and supplementary material.

Acknowledgments: The authors thank Eunkyung Kim and Jin-Hee Lee for data collection and management, and Hoon Suh for sample collection.

Conflicts of Interest: The authors have no conflict of interest to declare.

\title{
References
}

1. Siegel, R.L.; Miller, K.D.; Jemal, A. Cancer statistics, 2016. CA Cancer J. Clin. 2016, 66, 7-30. [CrossRef]

2. Tong, M.; Jun, T.; Nie, Y.; Hao, J.; Fan, D. The Role of the Slit/Robo Signaling Pathway. J. Cancer 2019, 10, 2694-2705. [CrossRef] [PubMed]

3. Jiang, Z.; Liang, G.; Xiao, Y.; Qin, T.; Chen, X.; Wu, E.; Ma, Q.; Wang, Z. Targeting the SLIT/ROBO pathway in tumor progression: Molecular mechanisms and therapeutic perspectives. Ther. Adv. Med. Oncol. 2019, 11, 1758835919855238. [CrossRef]

4. Marlow, R.; Strickland, P.; Lee, J.S.; Wu, X.; PeBenito, M.; Binnewies, M.; Hinck, L. SLITs suppress tumor growth in vivo by silencing Sdf1/Cxcr4 within breast epithelium. Cancer Res. 2008, 68, 7819-7827. [CrossRef]

5. Yuasa-Kawada, J.; Kinoshita-Kawada, M.; Rao, Y.; Wu, J.Y. Deubiquitinating enzyme USP33/VDU1 is required for Slit signaling in inhibiting breast cancer cell migration. Proc. Natl. Acad. Sci. USA 2009, 106, 14530-14535. [CrossRef] [PubMed]

6. Chang, P.-H.; Hwang-Verslues, W.W.; Chang, Y.-C.; Chen, C.-C.; Hsiao, M.; Jeng, Y.-M.; Chang, K.-J.; Lee, E.Y.-H.; Shew, J.-Y.; Lee, W.-H. Activation of Robo1 signaling of breast cancer cells by Slit2 from stromal fibroblast restrains tumorigenesis via blocking PI3K/Akt/ $\beta$-catenin pathway. Cancer Res. 2012, 72, 4652-4661. [CrossRef]

7. Dallol, A.; Morton, D.; Maher, E.R.; Latif, F. SLIT2 axon guidance molecule is frequently inactivated in colorectal cancer and suppresses growth of colorectal carcinoma cells. Cancer Res. 2003, 63, 1054-1058. [PubMed]

8. Huang, Z.; Wen, P.; Kong, R.; Cheng, H.; Zhang, B.; Quan, C.; Bian, Z.; Chen, M.; Zhang, Z.; Chen, X.; et al. USP33 mediates Slit-Robo signaling in inhibiting colorectal cancer cell migration. Int. J. Cancer 2015, 136, 1792-1802. [CrossRef] [PubMed]

9. Shi, R.; Yang, Z.; Liu, W.; Liu, B.; Xu, Z.; Zhang, Z. Knockdown of Slit2 promotes growth and motility in gastric cancer cells via activation of AKT/ $\beta$-catenin. Oncol. Rep. 2014, 31, 812-818. [CrossRef]

10. Xia, Y.; Wang, L.; Xu, Z.; Kong, R.; Wang, F.; Yin, K.; Xu, J.; Li, B.; He, Z.; Wang, L.; et al. Reduced USP33 expression in gastric cancer decreases inhibitory effects of Slit2-Robo1 signalling on cell migration and EMT. Cell Prolif. 2019, 52, e12606. [CrossRef]

11. Stella, M.C.; Trusolino, L.; Comoglio, P.M. The Slit/Robo system suppresses hepatocyte growth factor-dependent invasion and morphogenesis. Mol. Biol. Cell 2009, 20, 642-657. [CrossRef] [PubMed]

12. Lu, C.; Ning, Z.; Wang, A.; Chen, D.; Liu, X.; Xia, T.; Tekcham, D.S.; Wang, W.; Li, T.; Liu, X.; et al. USP10 suppresses tumor progression by inhibiting mTOR activation in hepatocellular carcinoma. Cancer Lett. 2018, 436, 139-148. [CrossRef]

13. Tseng, R.C.; Lee, S.H.; Hsu, H.S.; Chen, B.H.; Tsai, W.C.; Tzao, C.; Wang, Y.C. SLIT2 attenuation during lung cancer progression deregulates beta-catenin and E-cadherin and associates with poor prognosis. Cancer Res. 2010, 70, 543-551. [CrossRef]

14. Kong, R.; Yi, F.; Wen, P.; Liu, J.; Chen, X.; Ren, J.; Li, X.; Shang, Y.; Nie, Y.; Wu, K.; et al. Myo9b is a key player in SLIT/ROBOmediated lung tumor suppression. J. Clin. Investif. 2015, 125, 4407-4420. [CrossRef] [PubMed]

15. Sun, J.; Li, T.; Zhao, Y.; Huang, L.; Sun, H.; Wu, H.; Jiang, X. USP10 inhibits lung cancer cell growth and invasion by upregulating PTEN. Mol. Cell Biochem. 2018, 441, 1-7. [CrossRef] [PubMed]

16. Wang, Y.; Zhang, S.; Bao, H.; Mu, S.; Zhang, B.; Ma, H.; Ma, S. MicroRNA-365 promotes lung carcinogenesis by downregulating the USP33/SLIT2/ROBO1 signalling pathway. Cancer Cell Int. 2018, 18, 64. [CrossRef] [PubMed]

17. Jeon, M.J.; Lim, S.; You, M.-H.; Park, Y.; Song, D.E.; Sim, S.; Kim, T.Y.; Shong, Y.K.; Kim, W.B. The role of Slit2 as a tumor suppressor in thyroid cancer. Mol. Cell Endocrinol. 2019, 483, 87-96. [CrossRef]

18. Narayan, G.; Goparaju, C.; Arias-Pulido, H.; Kaufmann, A.M.; Schneider, A.; Dürst, M.; Murty, V.V. Promoter hypermethylationmediated inactivation of multiple Slit-Robo pathway genes in cervical cancer progression. Mol. Cancer 2006, 5, 16. [CrossRef] 
19. Beggs, A.D.; Jones, A.; El-Bahrawy, M.; Abulafi, M.; Hodgson, S.V.; Tomlinson, I.P. Whole-genome methylation analysis of benign and malignant colorectal tumours. J. Pathol. 2013, 229, 697-704. [CrossRef]

20. Dickinson, R.E.; Dallol, A.; Bieche, I.; Krex, D.; Morton, D.; Maher, E.R.; Latif, F. Epigenetic inactivation of SLIT3 and SLIT1 genes in human cancers. Br. J. Cancer 2004, 91, 2071-2078. [CrossRef]

21. Dallol, A.; Forgacs, E.; Martinez, A.; Sekido, Y.; Walker, R.L.; Kishida, T.; Rabbitts, P.H.; Maher, E.R.; Minna, J.D.; Latif, F. Tumour specific promoter region methylation of the human homologue of the Drosophila Roundabout gene DUTT1 (ROBO1) in human cancers. Oncogene 2002, 21, 3020-3028. [CrossRef]

22. Dammann, R.; Strunnikova, M.; Schagdarsurengin, U.; Rastetter, M.; Papritz, M.; Hattenhorst, U.E.; Hofmann, H.-S.; Silber, R.-E.; Burdach, S.; Hansen, G. CpG island methylation and expression of tumour-associated genes in lung carcinoma. Eur. J. Cancer 2005, 41, 1223-1236. [CrossRef]

23. Kwon, Y.-J.; Lee, S.J.; Koh, J.S.; Kim, S.H.; Lee, H.W.; Kang, M.C.; Bae, J.B.; Kim, Y.-J.; Park, J.H. Genome-wide analysis of DNA methylation and the gene expression change in lung cancer. J. Thorac. Oncol. 2012, 7, 20-33. [CrossRef] [PubMed]

24. Wang, J.; Yu, X.-F.; Ouyang, N.; Luo, Q.-L.; Zhao, S.-Y.; Guan, X.-F.; Chen, T.; Li, J.-X. Multi-platform analysis of methylationregulated genes in human lung adenocarcinoma. J. Toxicol. Environ. Health A 2019, 82, 37-45. [CrossRef]

25. Um, S.W.; Kim, H.K.; Kim, Y.; Lee, B.B.; Kim, D.; Han, J.; Kim, D.H. Bronchial biopsy specimen as a surrogate for DNA methylation analysis in inoperable lung cancer. Clin. Epigenet. 2017, 9, 131. [CrossRef]

26. Kim, J.S.; Han, J.; Shim, Y.M.; Park, J.; Kim, D.H. Aberrant methylation of H-cadherin (CDH13) promoter is associated with tumor progression in primary nonsmall cell lung carcinoma. Cancer 2005, 104, 1825-1833.

27. Edge, S.B.; Byrd, D.R.; Compton, C.C.; Fritz, A.G.; Greene, F.L.; Troth, A. American Joint Committee on Cancer. In AJCC Cancer Staging Manual, 7th ed.; Springer: New York, NY, USA, 2010; pp. 253-270.

28. Samejima, K.; Earnshaw, W.C. Trashing the genome: The role of nucleases during apoptosis. Nat. Rev. Mol. Cell Biol. 2005, 6, 677-688. [CrossRef] [PubMed]

29. Ivanov, M.; Baranova, A.; Butler, T.; Spellman, P.; Mileyko, V. Non-random fragmentation patterns in circulating cell-free DNA reflect epigenetic regulation. BMC Genom. 2015, 16, S1. [CrossRef] [PubMed]

30. Kim, Y.; Bin Lee, B.; Kim, D.; Um, S.; Cho, E.Y.; Han, J.; Shim, Y.M.; Kim, D. Clinicopathological significance of RUNX1 in non-small cell lung cancer. J. Clin. Med. 2020, 9, 1694. [CrossRef]

31. Tseng, R.-C.; Chang, J.-M.; Chen, J.-H.; Huang, W.-R.; Tang, Y.-A.; Kuo, I.-Y.; Yan, J.-J.; Lai, W.-W.; Wang, Y.-C. Deregulation of SLIT2-mediated Cdc42 activity is associated with esophageal cancer metastasis and poor prognosis. J. Thorac. Oncol. 2015, 10, 189-198. [CrossRef]

32. Qin, F.; Zhang, H.; Ma, L.; Liu, X.; Dai, K.; Li, W.; Gu, F.; Fu, L.; Ma, Y. Low expression of Slit2 and Robo 1 is associated with poor prognosis and brain-specific metastasis of breast cancer patients. Sci. Rep. 2015, 5, 14430. [CrossRef]

33. Liu, L.; Li, W.; Geng, S.; Fang, Y.; Sun, Z.; Hu, H.; Liang, Z.; Yan, Z. Slit2 and Robo1 expression as biomarkers for assessing prognosis in brain glioma patients. Surg. Oncol. 2016, 25, 405-410. [CrossRef] [PubMed]

34. Mohamed, G.; Talima, S.; Li, L.; Wei, W.; Rudzki, Z.; Allam, R.M.; Simmons, W.; Tao, Q.; Murray, P.G. Low expression and promoter hypermethylation of the tumour suppressor SLIT2, are associated with adverse patient outcomes in diffuse large B cell lymphoma. Pathol. Oncol. Res. 2019, 25, 1223-1231. [CrossRef]

35. Zhang, C.; Guo, H.; Li, B.; Sui, C.; Zhang, Y.; Xia, X.; Qin, Y.; Ye, L.; Xie, F.; Wang, H.; et al. Effects of Slit3 silencing on the invasive ability of lung carcinoma A549 cells. Oncol. Rep. 2015, 34, 952-960. [CrossRef] [PubMed]

36. Risberg, B.; Tsui, D.W.; Biggs, H.; de Almagro, A.R.-V.M.; Dawson, S.-J.; Hodgkin, C.; Jones, L.; Parkinson, C.; Piskorz, A.; Marass, F.; et al. Effects of collection and procession on plasma circulating cell-free DNA from cancer patients. J. Mol. Diagn. 2018, 20, 883-892. [CrossRef] [PubMed]

37. Kerachian, M.A.; Azghandi, M.; Mozaffari-Jovin, S.; Thierry, A.R. Guidelines for pre-analytical conditions for assessing the methylation of circulating cell-free DNA. Clin. Epigenet. 2021, 13, 193. [CrossRef] 